Supporting Information

\title{
Rapid detection of SARS-CoV-2 antigens and antibodies using OFET biosensors based on a soft and stretchable semiconducting polymer
}

Kristina Ditte ${ }^{1,2} \neq$, Trang Anh Nguyen Le $e^{3,4} \neq$, Oliver Ditzer ${ }^{1,2}$, Diana Isabel Sandoval Bojorquez $z^{3}$, Soosang Chae ${ }^{1}$, Michael Bachmann ${ }^{3,4}$, Larysa Baraban ${ }^{3 *}$, and Franziska Lissel ${ }^{1,2,5 *}$.

${ }^{1}$ Leibniz Institute of Polymer Research Dresden e.V., Hohe Straße 6, Dresden 01069, Germany;

${ }^{2}$ Faculty of Chemistry and Food Chemistry, Dresden Technical University, Dresden 01062, Germany;

3 Helmholtz-Zentrum Dresden-Rossendorf e.V., Institute of Radiopharmaceutical Cancer Research, Bautzner Landstrasse 400, 01328 Dresden, Germany;

${ }^{4}$ Faculty of Medicine Carl Gustav Carus, Dresden Technical University, Dresden 01307, Germany;

${ }^{5}$ Institute of Organic Chemistry and Macromolecular Chemistry, Friedrich Schiller University Jena, Humboldtstr. 10, 07743 Jena, Germany.

Correspond to Dr. Franziska Lissel (Email: lissel@ipfdd.de)

Dr. Larysa Baraban (Email: 1.baraban@thzdr.de)

Keywords: organic field-effect transistors, biosensors, block copolymers, semiconducting polymers, SARS-CoV-2 antigen sensor, anti-SARS-COV-2 antibodies sensor. 

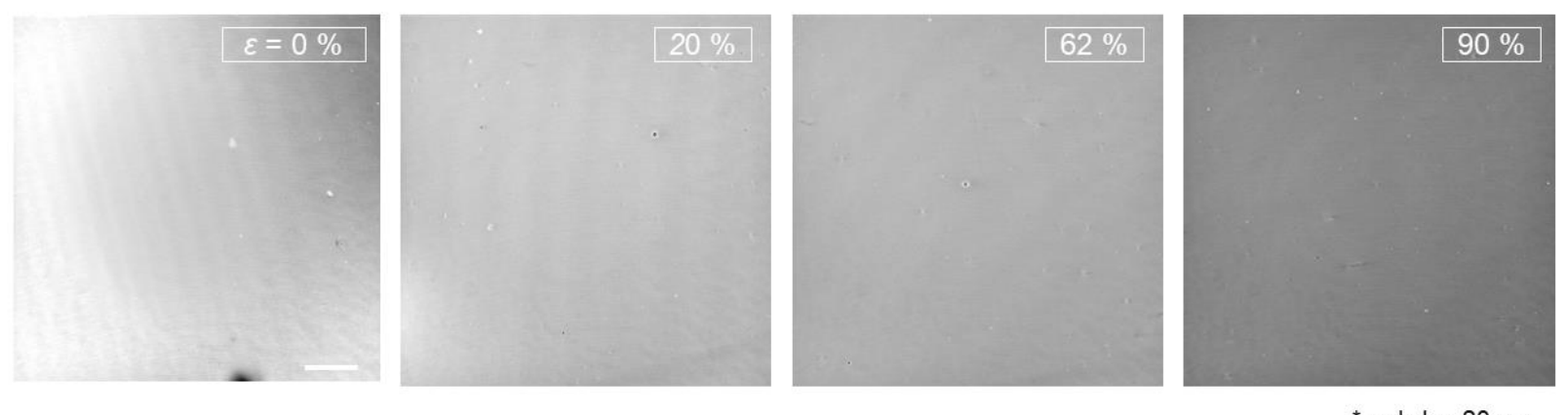

*scale bar $20 \mu \mathrm{m}$
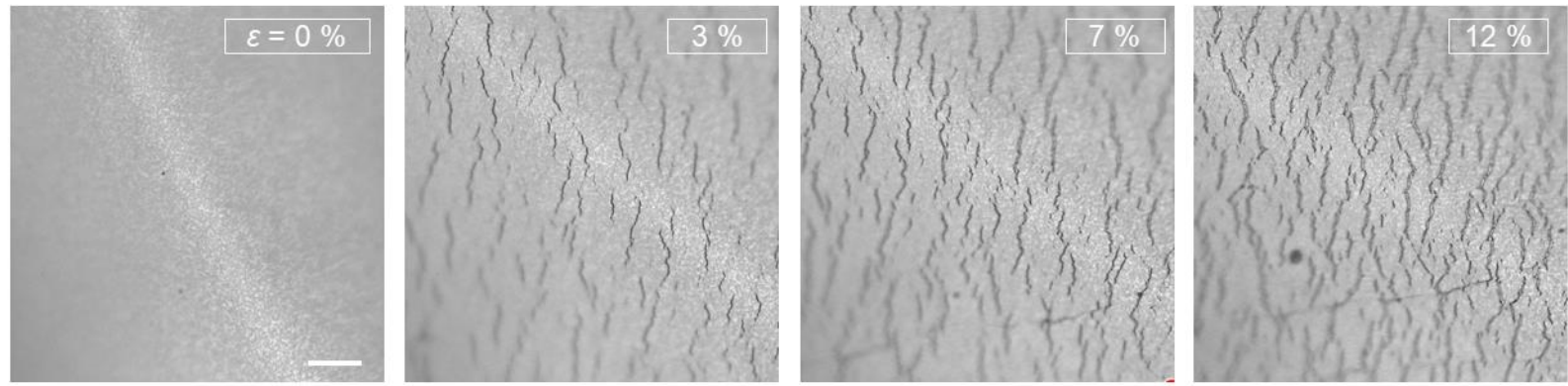

*scale bar $50 \mu \mathrm{m}$

Figure S1. Microscopy images of strained TBC (upper) and reference PDPP-TT (lower), respectively (scale bars denote $20 \mu \mathrm{m}$ for TBC and $50 \mu \mathrm{m}$ for the reference PDPP-TT, and $\varepsilon$ is the amount of applied strain). Note: there is a technical limitation to apply strain of more than $90 \%$ on the film, therefore this value is reported as the final one. 

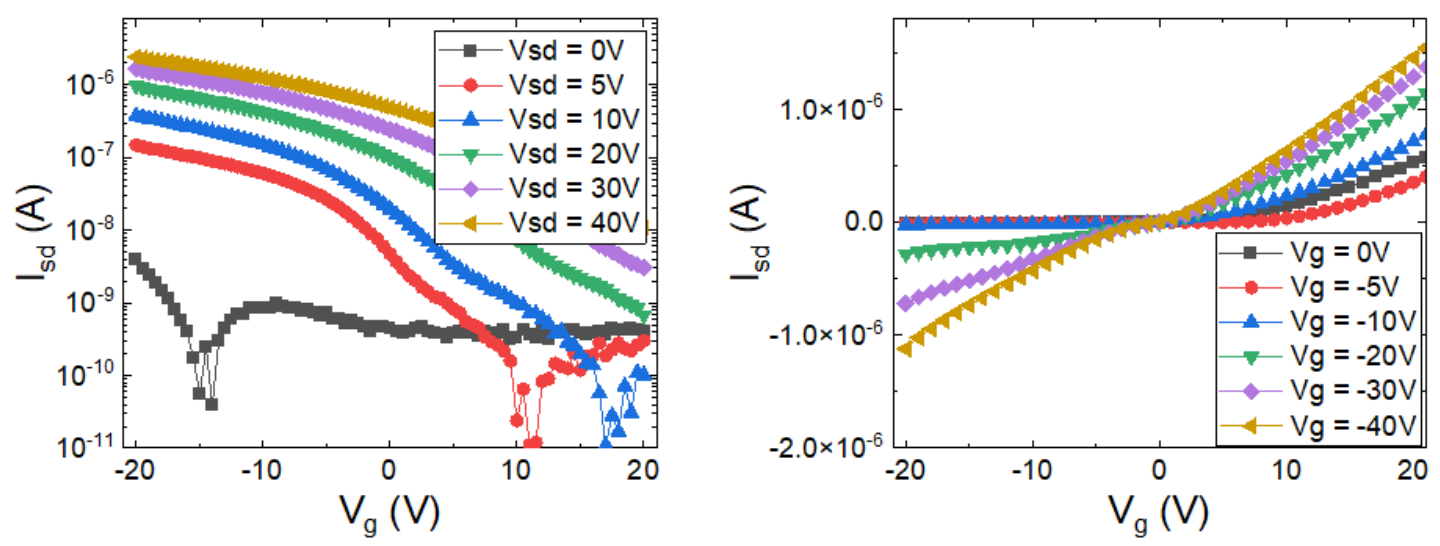

Figure S2. (A) Transfer and (B) output electrical characteristics of PDPP-TT OFET. 

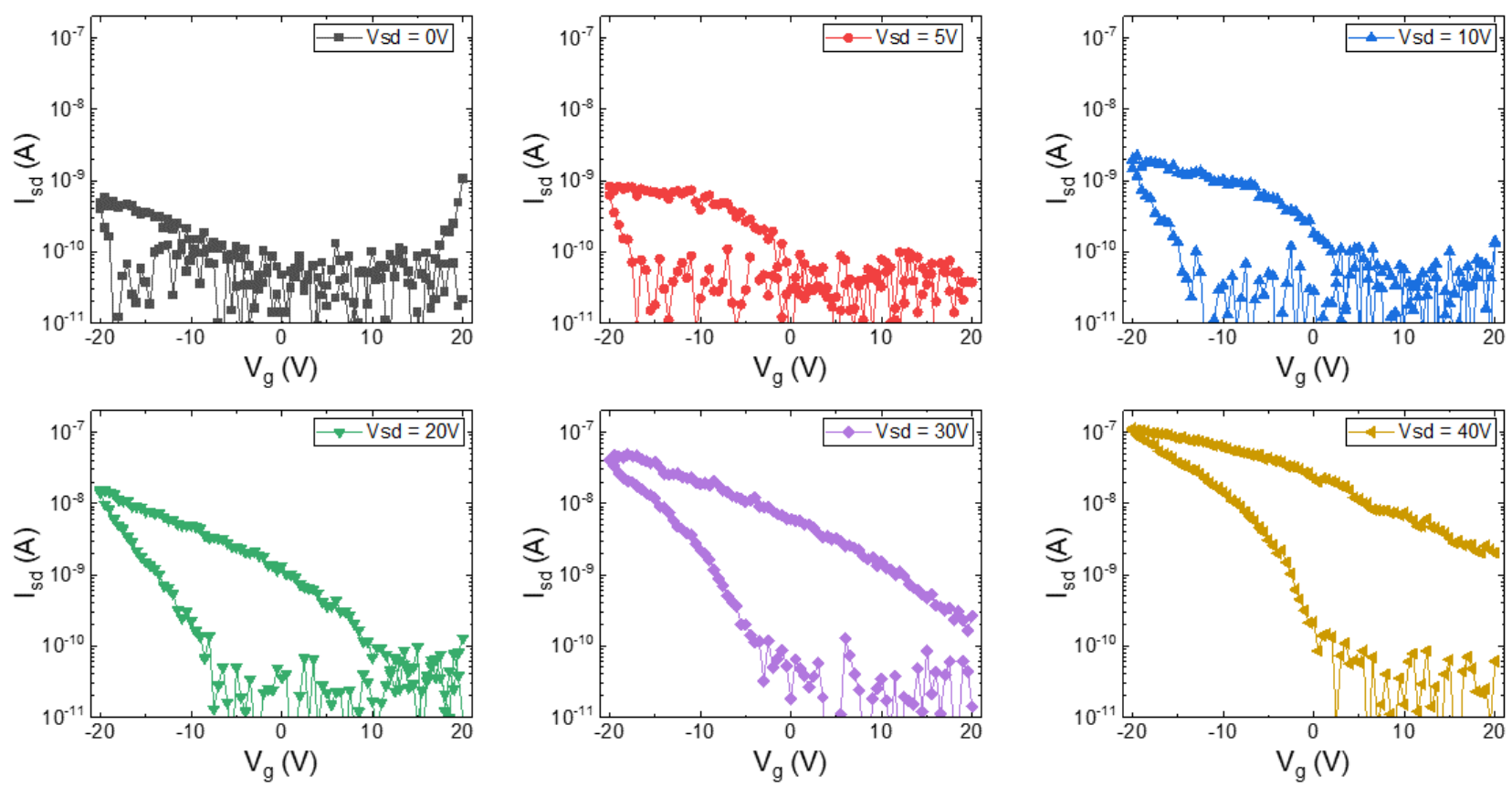

Figure S3. Transfer characteristics (forward and backward sweep) of pristine TBC-based OFET device at different $\mathrm{V}_{\text {sd. }}$ 

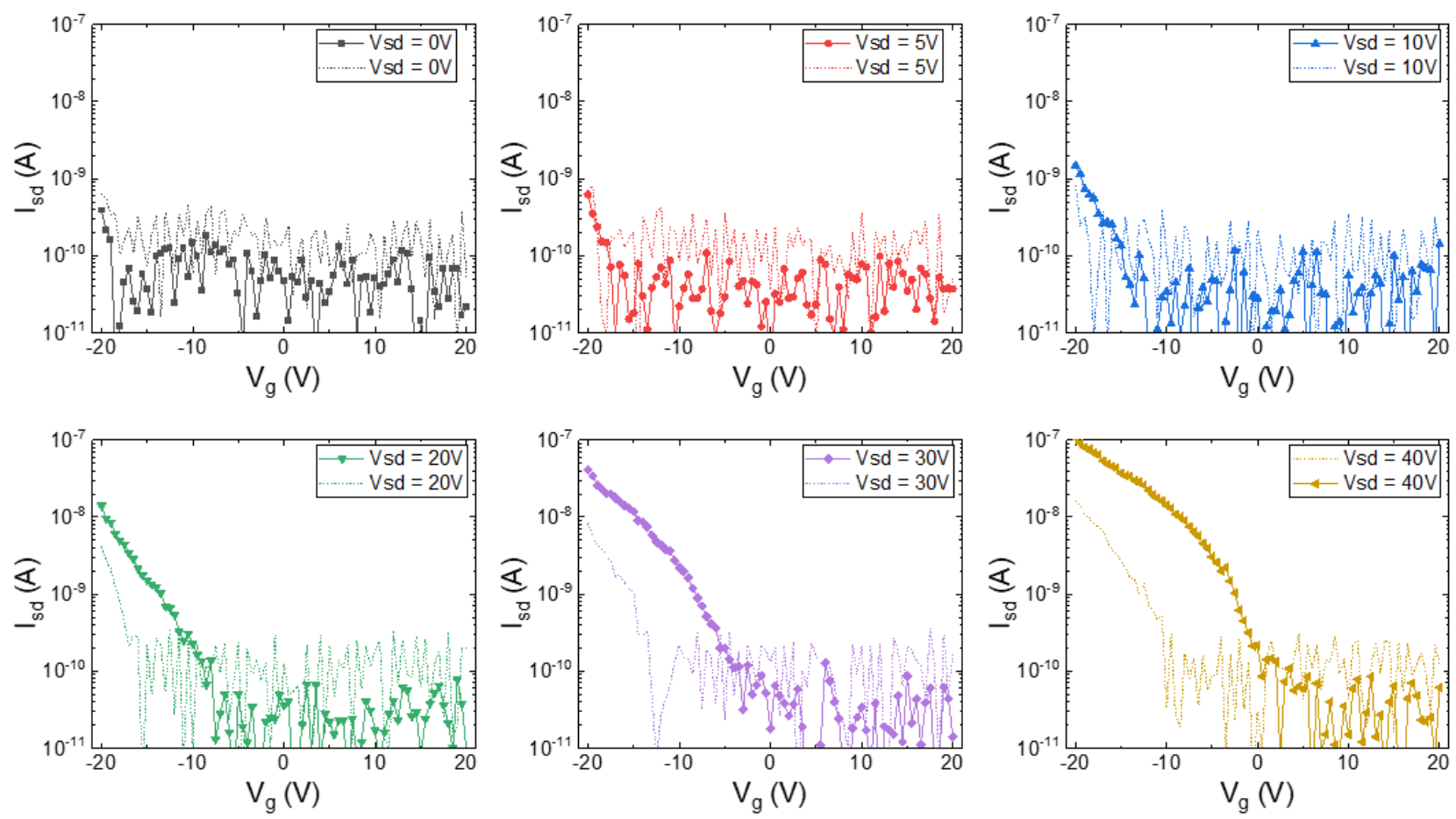

Figure S4. Transfer characteristics (forward sweep) of bare TBC-based OFET device at different $\mathrm{V}_{\text {sd }}$ and measured leakage current (dashed line). 

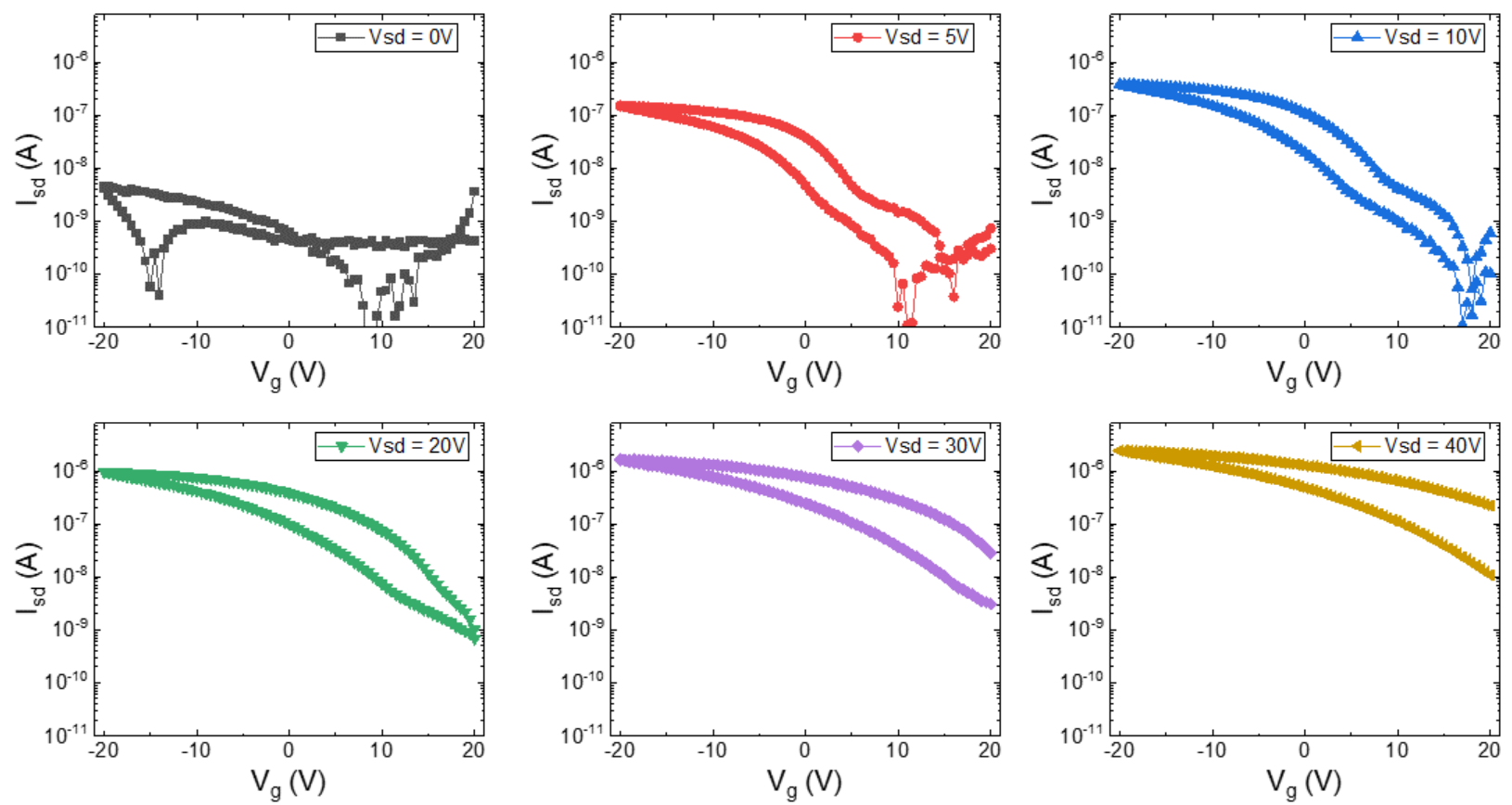

Figure S5. Transfer characteristics (forward and backward sweep) of bare reference PDPP-TTbased OFET device at different $\mathrm{V}_{\text {sd. }}$. 

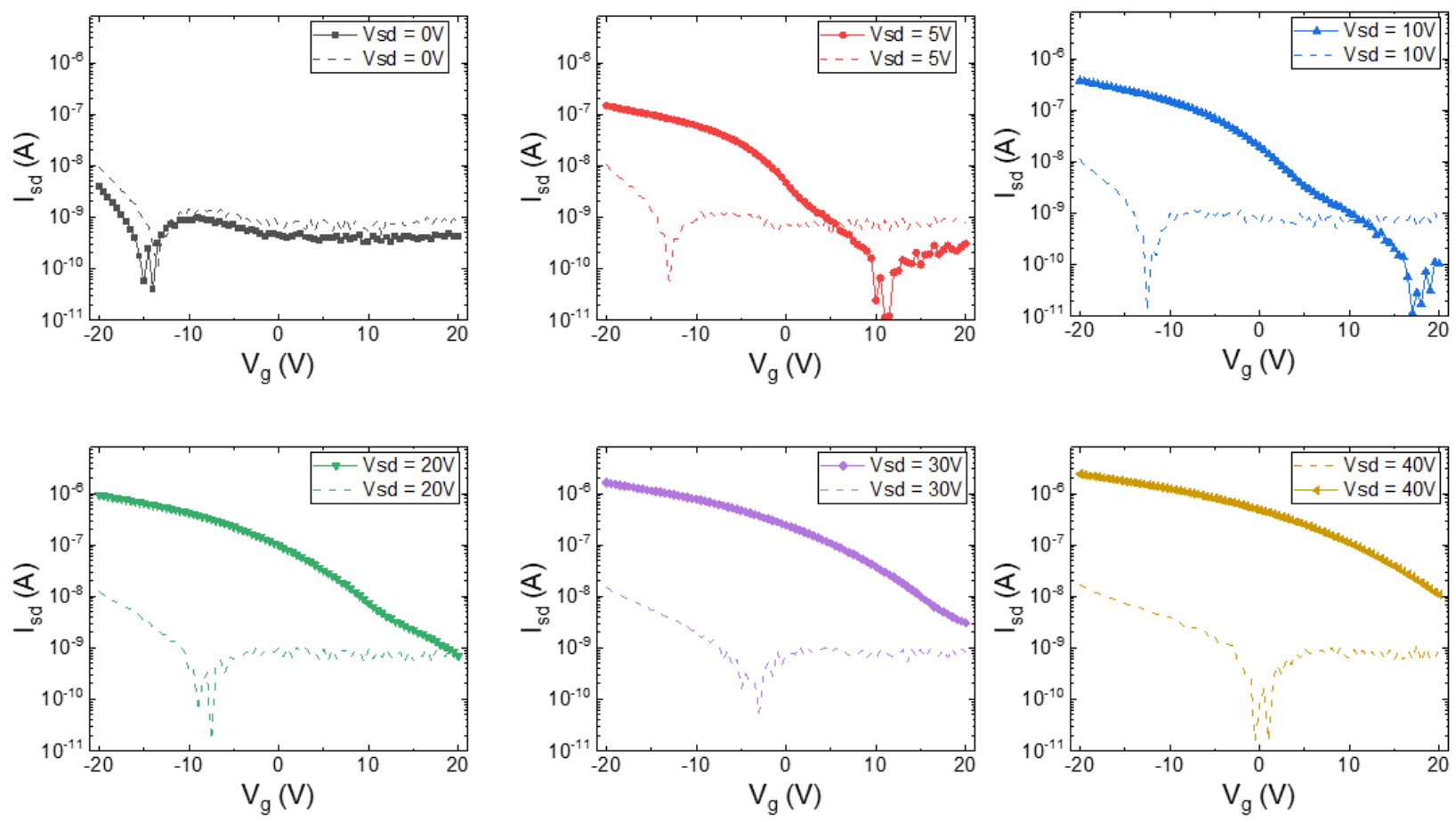

Figure S6. Transfer characteristics (forward sweep) of bare reference PDPP-TT-based OFET device at different $\mathrm{V}_{\text {sd }}$ and measured leakage current (dashed line). 
A.

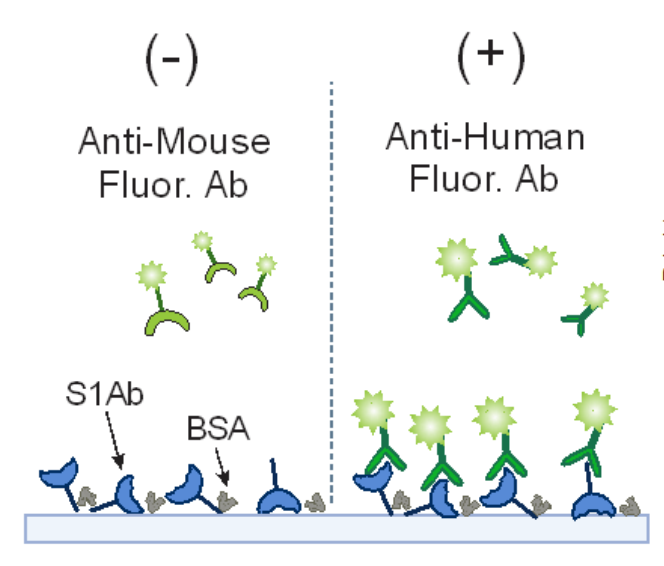

B.

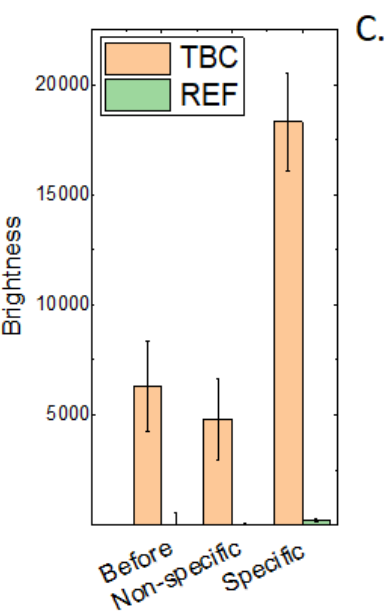

C.

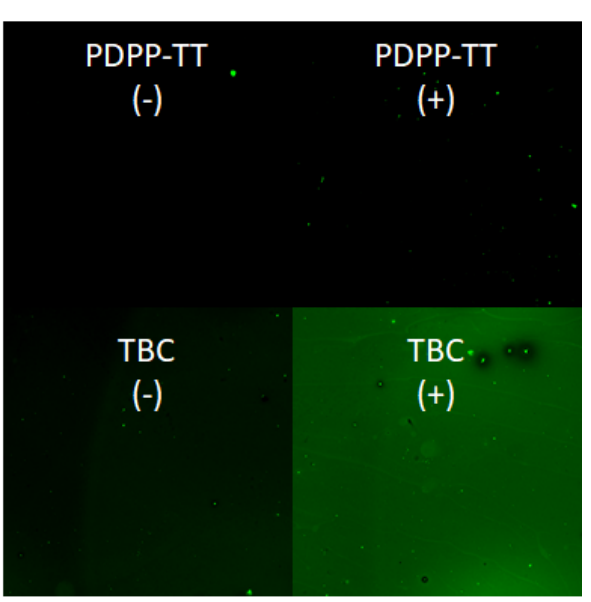

Figure S7. Verification of S1Ab binding to TBC and non-specific adsorption. (A) Concept illustration: TBC/PDPP-TT polymer sample functionalized with S1Ab and BSA blocking were exposed to anti-mouse fluorescence secondary antibodies for non-specific adsorption testing (-) and anti-human fluorescence secondary antibodies for verifying presence of S1Ab (+). (B) Brightness of fluorescence signal measured from fluorescence images of the samples under different condition. (C) Fluorescence microscopy images of the samples. Overall, TBC expressed certain level of auto-fluorescence with low non-specific binding and higher amount of S1Ab bound to the surface than PDPP-TT reference sample. 
A.

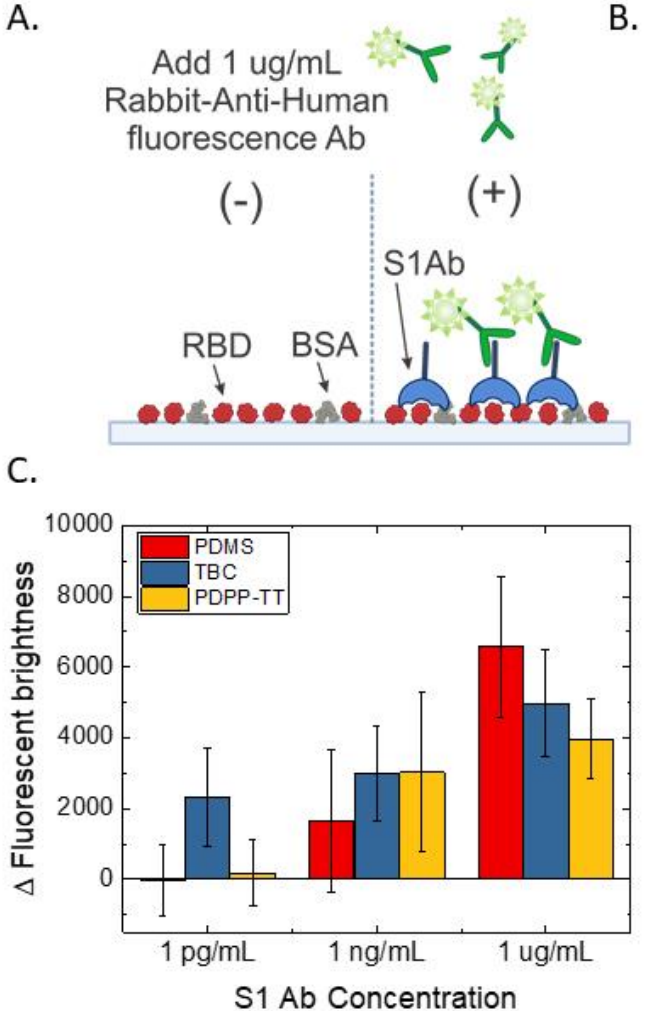

C.
B.

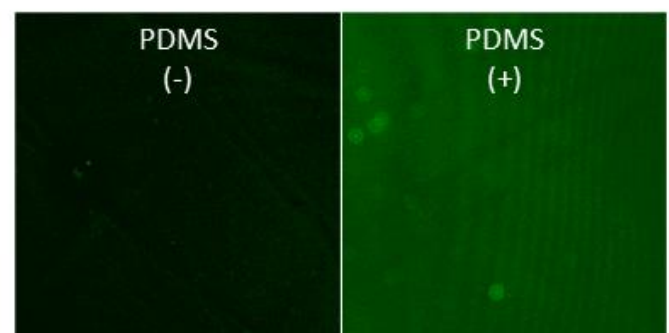

PDPP-TT

$(-)$

PDPP-TT

$(+)$

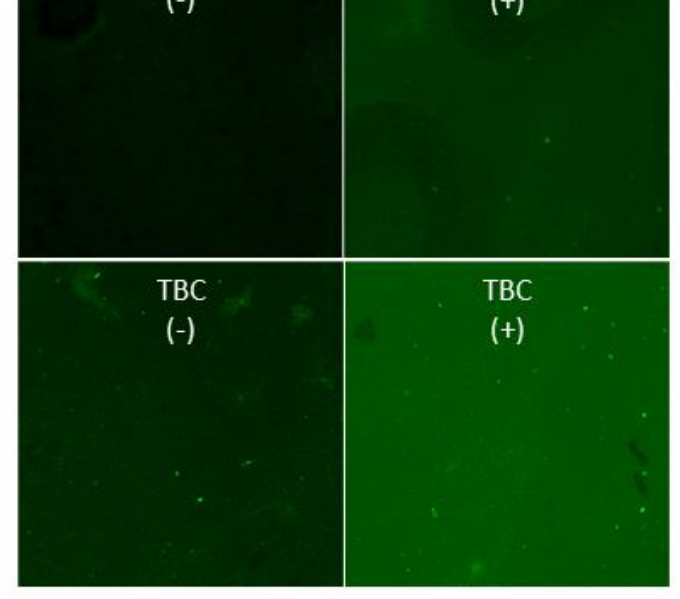

Figure S8. Confirmation of S1 Abs binding event by utilization of fluorescence signal measurement: (A) Concept illustration: secondary fluorescent antibodies are added to PDMS/ reference PDPP-TT/TBC surface functionalized with BSA, RBD for the negative (-) control samples, and addition of S1Ab as positive $(+)$ control sample. As secondary Rabbit-anti-Human antibodies have good affinity to $\mathrm{Hu}-\mathrm{S} 1 \mathrm{Ab}$, only positive $(+)$ control samples are expected to express fluorescent signal. (B) Fluorescence images of PDMS/ reference PDPP-TT/TBC substrates in negative (-) and positive (+) control samples $(1 \mu \mathrm{g} / \mathrm{mL})$. (C) The obtained brightness of the fluorescence subtract to negative (-) control samples measured for different S1 Abs solution concentrations. 
A.

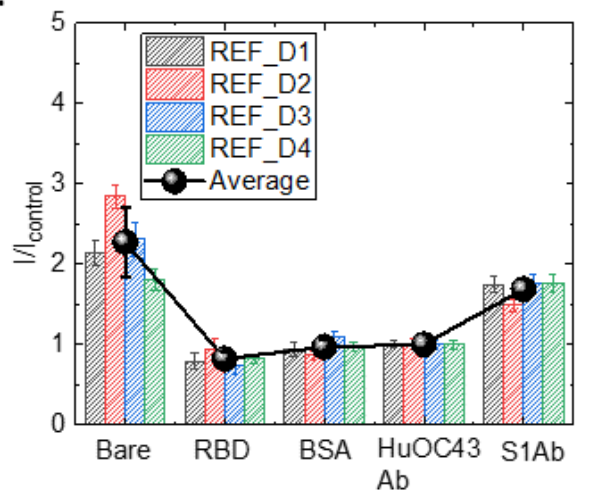

C.

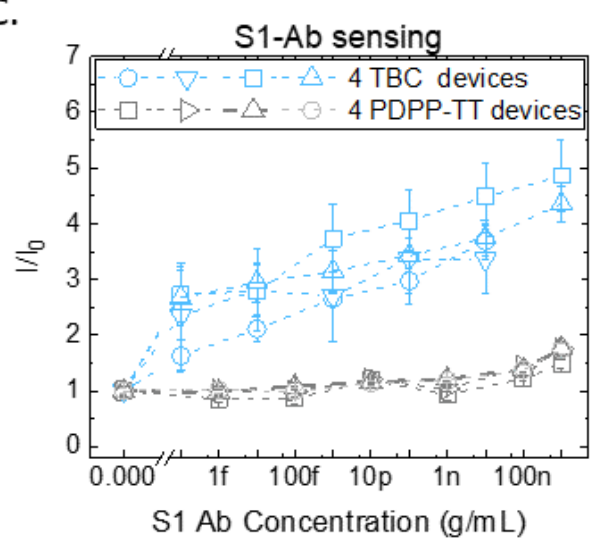

B.

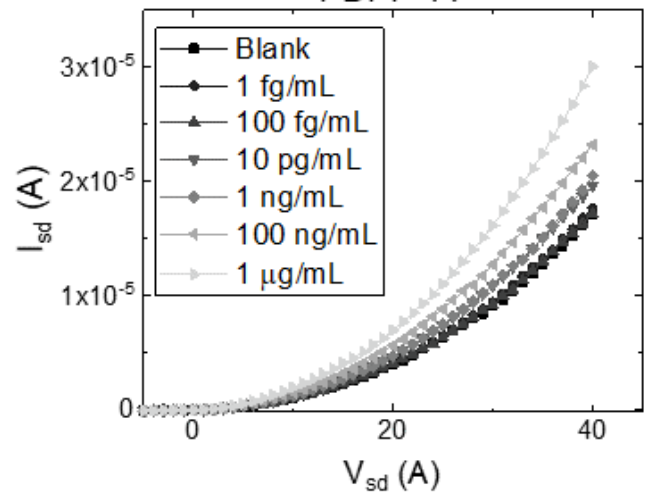

D.

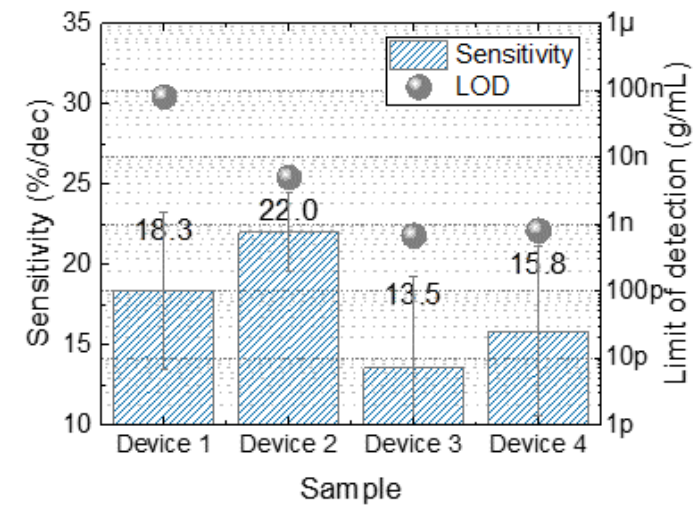

Figure S9. Reference PDPP-TT-based OFET sensing of S1 Abs: (A) Comparison in output current at $\mathrm{V}_{\mathrm{g}}=-20 \mathrm{~V}$ and $\mathrm{V}_{\mathrm{sd}}=40 \mathrm{~V}$ for 4 OFETs after each modification step. Error bars present stand ard deviation of five measurements. (B) S1 Abs concentration dependence of output characteristics (from $1 \mathrm{fg} / \mathrm{mL}$ to $1 \mathrm{um} / \mathrm{mL}$ ). (C) Comparison of current ratio to blank sample in TBC-based OFET (blue) and PDPP-TT-based OFET (gray). Error bars represent standard deviation of five measurements. (D) Obtained sensitivity and LOD of the reference PDPPTT-based OFET: mean LOD $3.8 \mathrm{ng} / \mathrm{mL}$, sensitivity $17.4 \% / \mathrm{dec}$. 

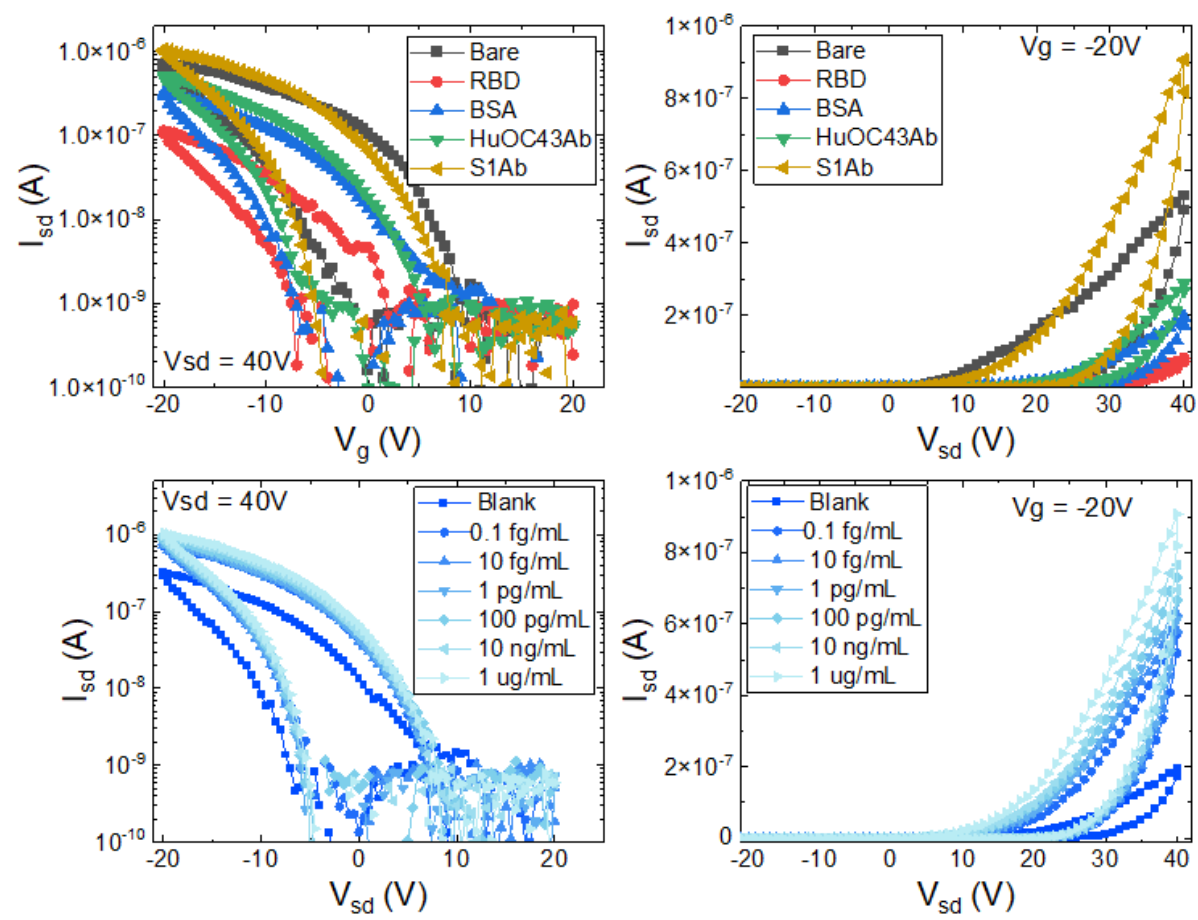

Figure S10. (A-C) Transfer and (B-D) output electrical characteristics of TBC-based

OFET in antibody sensing strategy (A-B) after each surface modification stepand (C-D) after incubation with different concentration of S1 antibodies. 


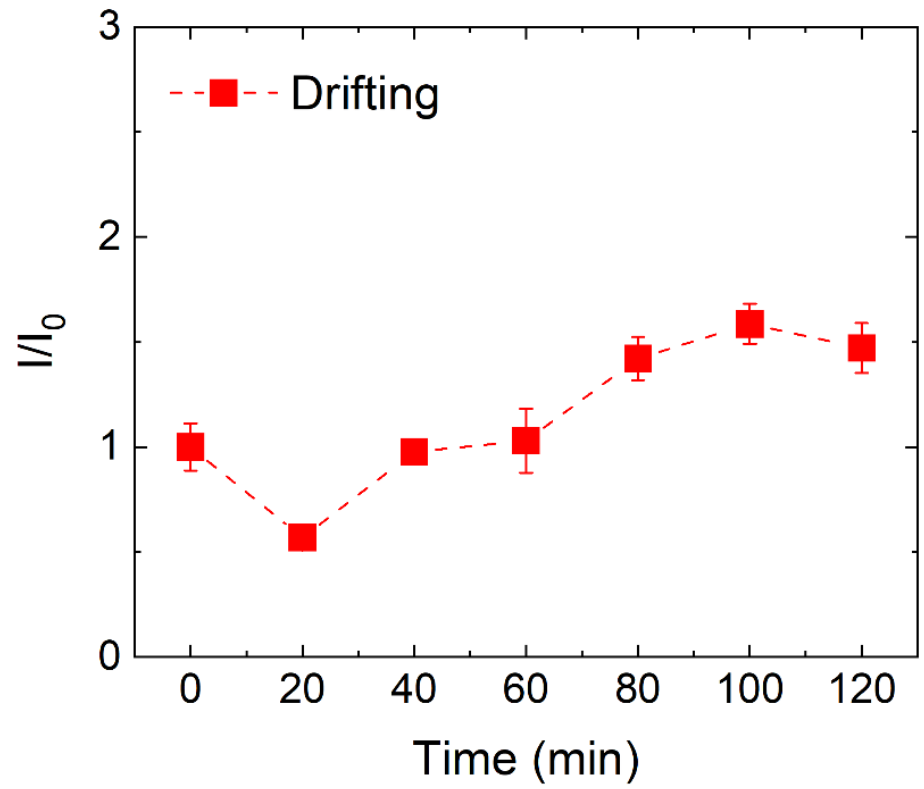

Figure S11. Drift current measurements: extracted output current ratio during the electrical measurements of TBC-based OFET for the duration of every 20 minutes over two hours. 
RBD sequence:

RVQPTESIVRFPNITNLCPFGEVFNATRFASVYAWNRKRISNCVADYSVLYNSASFSTFK CYGVSPTKLNDLCFTNVYADSFVIRGDEVRQIAPGQTGKIADYNYKLPDDFTGCVIAWN SNNLDSKVGGNYNYLYRLFRKSNLKPFERDISTEIYQAGSTPCNGVEGFNCYFPLQSYGF QPTNGVGYQPYRVVVLSFEL LHAPATVCGPKKSTNLVKNKCVNF 